\title{
Smart Advances in Healthcare for Geriatric Patients
}

\author{
Thomas W Miller* \\ Department of Gerontology, College of Public Health, University of Kentucky, UK \\ *Corresponding author: Thomas W Miller, Department of Gerontology, College of Public Health, Department of Psychiatry, College \\ Medicine, University of Kentucky. UK \\ To Cite This Article: Miller Thomas W. Smart Advances in Healthcare for Geriatric Patients. Am J Biomed Sci \& Res. 2019 - 1(3). AJBSR. \\ MS.ID.000526. DOI: 10.34297/AJBSR.2019.01.000526
}

Received: February 09, 2019 | Published: February 15, 2019

\section{Review}

Biomedical science is recognizing the value and importance of applications utilizing SMART technologies in the care and treatment of our aging population. Improved health and social care over recent years has increased life expectancy worldwide. As a result, nearly $7 \%$ of the world's population is now over 65 years of age. The proportion of older people is predicted to rise approximately $20 \%$ by 2050 worldwide [1]. The increasing number and proportion of older adults requires a greater focus on policies and resources to meet the needs of this growing global population. Smart home technologies encourage and allow aging individuals to live longer in their own homes.

When we speak of this technology, SMART stands for SelfMonitoring Analysis and Reporting Technology. Recent clinical research [2-5] has identified the value and benefits of wearable SMART technology for various populations raising the question as to whether there may be beneficial use with individuals in treatment for health-related conditions. Wearable SMART technology employs digital technologies to collect health data from individuals in one location, such as a patient's home, and electronically transmits the information to healthcare providers in a different location like a primary health care center for assessment, monitoring and compliance [3].

In consideration of these applications there are several home systems and devices that can enable seniors to live in their own homes longer. Seniors have benefited from remote monitoring types of technology to aid them in having quick access to emergency networks. Seniors with early stage Alzheimer's Syndrome or similar neurological conditions have benefited from these automated home features. These devices can help foster a safe and independent living environment. Personal wearable SMART technology devices are already available and the subject of research beneficial to a growing geriatric population. Among these is the Apple "iWatch" which offers close to a dozen different sensors, tracking the wearers' health and fitness metrics in terms of steps taken, calories burned, blood glucose, sleep quality, blood pressure and heart rate. There are several customized services available for families who have a senior family member needing monitoring. Applications to create a "SMART Medical Home" [6] will impact the care and treatment of seniors and aid caregivers who may benefit from computer based technology in their homes to provide necessary monitoring of behavior and maintain health and wellness for the aging population worldwide.

Specialists in the aging community are engaging biomedical professionals in recognizing the value and benefits of utilizing SMART tech $\neg$ nology options in providing clinical care [7]. E-health, which is the use of telemedicine and telecommunications for healthcare practice, is utilizing technology-based diagnostic and treatment strategies that have the potential to deliver benefits to a broad range of individuals who experience complex medical conditions $[3,5,8]$. SMART technology is a form of synchronous or asynchronous healthcare delivery that may serve as an adjunct to standardized forms of clinical treatment intervention. Contemporary models of medically supervised e-healthcare include integrated clinical models that emphasize multidisciplinary teams treating patients. Allied health multidisciplinary professionals are recognized as bringing specialized skills to the integrated health care teams. In treating the whole person, contemporary care often requires an integrated team approach that addresses both physical and mental health components involving intervention models that require a limited number of appointments that demonstrate mea surable improvements in the current managed care environment. Wearable SMART technology provides a potential adjunct for use in assessing the therapeutic progress of various cognitive and behavioral activities of patients.

University research centers along with governmental agencies are strongly encouraged to further support greater joint efforts of scientists and engineers from multiple sites internationally to 
frame our expanding knowledge and evidence-based research studies for SMART healthcare on a multidisciplinary level. For those who have been effected by cognitive changes, neuro-restoration involves lost neurological function due to the traumatic or ischemic damage to the brain and the associated neurological deficits. SMART technology can contribute to the rehabilitation of traumatic brain injury and stroke. SMART sensor-enabled technologies and implants have provided real-time information to medical professionals for positioning and post-operative evaluation for better patient care throughout the treatment pathway. These implants have the potential to reduce periprosthetic infection, which is a serious problem in clinical practice.

Within the SMART medical home, a computer can track the location of pre-programmed items such as eyeglasses, coffee cups, or car keys throughout the house so an aging person with cognitive deficits can locate such items when needed. The SMART technology can aid the person in restoring some lost skills due to brain injury. A camera-based scanning system can take three-dimensional textured images of one's skin, automatically checking for carefully watched skin features such as moles or skin irritation. Other SMART trackers involve monitors that use cameras located around the home.

When a picture of the sought-after item is touched on a monitor, the system pinpoints where the item was last recorded, an especially helpful tool for impaired aging individual due to dementia.

Gait monitors have been developed to track the way the aging person ambulates on various surfaces and in different locations. This SMART system's computers make comparisons over time, checking for any indications of shuffling or limping that may be the precursor to further physical impairment. It is also especially helpful for detecting symptoms of a stroke or for the trembling that may accompany Parkinson's disease. By identifying these signs and symptoms early, the hope is that a disease's full effects can be prevented or ameliorated.

Utilization of SMART technology for health and wellness can be incorporated into one's tool kit along with an effective diet and exercise program can make all the difference for these patients in achieving improved and recommended health goals in-cluding weight, diet, sleep, exercise, self-esteem and an improved quality of life. The use of wearable technology devices like the popular Fitbit has become an enormously popular health \& fitness gauge for about a decade. Health and wellness prone individuals' benefit from self-tracking using such a device as they provide a variety of self-monitoring information and biomarkers.

Multiple research efforts have used different terminology to capture the essence of SMART based e-health and the overall language used to describe the benefits of wearable SMART technologies. There is clear evidence that the utility and benefits of such technology is being established and beneficial to aging individuals [5,9]. SMART technology advances for improving healthcare delivery, wearable appliances that form an integral part of our personal life have emerged. The use of $21^{\text {st }}$ century technology is shaping the delivery of biomedical clinical care for geriatric patients. On the positive side, technology's integration with specific counseling skills is inevitable and show favorable potential to assist with improved patient self-management. Because of the early investigations into this modality, single case study design has served as a pilot level research for evidence-based decision making with SMART technology. With the use of case study designs, there is the realization that there are a number of limitations. Lacking are certain models or analyses that might include a multiple subject design models and randomization to assess the ef-fectiveness of multiple models of the SMART technology. What it represents is an introduction to the use of SMART based research for wearable tech $\neg$ nology in monitoring and promoting self-monitoring among aging patients who experience a medical condition that could benefit from life style changes.

SMART technology incorporated into the collaborative efforts of biomedical science and practice clinicians provides a significant pathway in the prevention, early detection, and treatment intervention strategies for geriatric patients [5]. The use of SMART technology in self-monitoring compliance, health, wellness and behavior change with patients motivated to utilize SMART technology in complying with prescribed clinical treatment continues to contribute to biomedical science.

\section{References}

1. (2001) Organization for Economic Co-operation and Development [OECD] (2001) World Population and Ageing, Annual Conference, Paris, France.

2. Miller TW, Kraus RF, Standafer V (2017) SMART Technology: Redefining Geriatric Health Care. International Journal of Geriatrics \& Gerontology IJGG-103. 4 (1): 1-7.

3. Allard M, Husky M, Catheline G, Pelletier A, Dilharreguy B, et al. (2014) Mobile technologies in the early detection of cognitive decline. PLoS One23: 9(12).

4. De Lucia PR, Harold SA, Tang YY (2013) Innovation in technology-3 Aided psychotherapy through human factors/ergonomics: Toward a collaborative approach. Journal of Contemporary Psychotherapy 43(2): 253-260.

5. Bashshur RL, Howell JD, Krupinski KA, Harms KM, Bashshur N, et al. (2016) The Empirical Foundations of Telemedicine Interventions in Primary Care. Telemed J E Health 22(5): 342-375.

6. Carabalona R, Grossi F, Tessadri A, Castiglioni P, Caracciolo A, et al. (2012) Light on! Real world evaluation of a P300-based brain-computer interface $(\mathrm{BCI})$ for environment control in a smart home. Ergonomics 55(5): 552-563.

7. Rosenberg L, Kottorp A, Nygard L (2011) Readiness for Technology Use with People with Dementia: The Perspectives of Significant Others. Journal of Applied Gerontology 31(4): 510-530.

8. Miller TW, Wood J (2012) Telepractice: A 21st Century Model of Health Care Delivery. In: Guah, Matthew (Ed.), Healthcare Delivery: Reform and New Technologies, Information Science Publishers, New York, USA.

9. Chan M, Esteve D, Fourniols JY, Escriba C, Campo E (2012) Smart 6. Wearable systems: Current status and future challenges. Artif Intell Med 56(3): 137-156. 